\title{
Fortalecimiento de las capacidades de diagnóstico e investigación del Laboratorio de Salud Pública de la Facultad de Ciencias Exactas (UNLP), integrado a la red de diagnóstico de SARS-CoV-2 de la Provincia de Buenos Aires
}

\author{
Guillermo H Docena*, Grupo COVID Exactas ${ }^{1}$ \\ Facultad de Ciencias Exactas, Universidad Nacional de La Plata*; E-mail:
}

guillermo.docena@gmail.com

${ }^{1}$ Al final del resumen se presenta la lista de participantes del grupo y las Unidades Ejecutoras involucradas.

Resumen. Desde que se informó el primer caso de enfermedad por COVID-19 en China, en diciembre de 2019, y se declaró la crisis sanitaria y luego la pandemia, los sistemas de Salud debieron adaptar sus estructuras para realizar diagnóstico, vigilancia, monitoreo de la respuesta inmune y evaluación de la protección inmune post-infección o post-vacunación. En este escenario el Grupo COVID Exactas, a través del Laboratorio de Salud Pública, de la Secretaría de Extensión de la Facultad de Ciencias Exactas, montó un laboratorio de diagnóstico para realizar la detección del RNA viral en muestras de pacientes mediante su amplificación RT-qPCR. Para paliar la falta de disponibilidad de reactivos y simplificar la extracción del RNA se encaró el desarrollo de un sistema basado en nanopartículas magnéticas recubiertas de sílice, que no requieren el uso de centrifuga. Al mismo tiempo, el grupo Serología comenzó a monitorear anticuerpos séricos IgG específicos, mientras que el grupo Investigación comenzó a desarrollar inmunoensayos para detección de IgG/IgM/IgA específicos y caracterización de espectros infrarrojo y fluorescente en muestras de orina. En este contexto de gravedad sanitaria la Universidad Nacional de La Plata (UNLP) y el Ministerio de Salud de la Provincia de Buenos Aires (PBA) firmaron un convenio de cooperación y trabajaron en la adecuación de laboratorios, coordinación de distribución de muestras, provisión de reactivos e insumos y diseño de estrategias conjuntas de vigilancia para aportar soluciones al sistema de salud en el contexto de pandemia. Asimismo, el interés del Gobierno de la PBA por los desarrollos de los institutos de la UNLP y de doble dependencia UNLPCONICET, dio lugar a otro convenio tripartito con finalidades específicas. 
Palabras Clave: SARS-CoV-2, COVID-19, diagnóstico RT-qPCR, ELISA, espectroscopía, RNA, antígenos, anticuerpos.

Recibido: 01/11/2020 Aceptado: 04/11/2020

DOI: https://doi.org/10.24215/26838559e017

\section{Fostering SARS-CoV-2 diagnosis and research capacity at the Public Health Laboratory in the School of Sciences of the University of La Plata}

Abstract. After the first COVID-19 case was reported in China in December 2019, leading to a sanitary crisis and later expanded to a pandemic, Public Health Systems were forced to adapt their facilities and capabilities to face, among other actions, the need for massive diagnostics, surveillance, monitoring immune response and assess protection post-infection and, eventually, after vaccination. In this scenario, many researchers, professionals, recent graduates and students at the School of Sciences (Facultad de Ciencias Exactas, Universidad Nacional de La Plata) assembled a multisdiciplinary team called "Grupo COVID Exactas". This group helped to set up a facility to perform routine RT-qPCR-based detection of viral RNA in clinical samples at the Laboratory of Public Health of the Secretary of Extension of the Faculty (Secretaría de Extension de la Facultad de Ciencias Exactas de la Universidad Nacional de La Plata).

To cope with the shortage of reagents worldwide and to simplify RNA isolation, a silica-coated magnetic bead-based system was developed locally to avoid centrifugation steps. At the same time, the serology group initiated specific IgG screening tests, and the research team addressed the development of immunoassays to detect SARS-CoV-2-specific IgG/IgM/IgA. Also, urine infrared and fluorescence spectra are being explored for their potential to find differential patterns associated with the disease. The seriousness of the public health problem led the signature of a cooperation agreement between the UNLP and the Ministry of Health of the Province of Buenos Aires (Ministerio de Salud de la Provincia de Buenos Aires) to update the laboratory setups, to coordinate distribution of clinical samples and laboratory and to provide with PPE supplies. It also includes the design and implementation of screening and surveillance strategies addressing issues 
associated with pandemics. In addition, the interest of the Government of the PBA in the specific local development of technologies and reagents at the University is the focus of another recently signed tripartite agreement that includes R\&D institutes of the UNLP also associated with CONICET (Argentinean National Research Council).

Key Words: SARS-CoV-2, COVID-19, RT-qPCR diagnosis, ELISA, spectroscopy, RNA, antigens, antibodies.

\section{Grupo COVID Exactas}

Participan del Grupo COVID Exactas miembros de diferentes unidades ejecutoras de la Facultad de Ciencias Exactas y otros voluntarios:

- $\quad$ IIFP (Instituto de Estudios Inmunológicos y Fisiopatológicos, UNLP-CONICET-CIC)

- $\quad$ IBBM (Instituto de Biotecnología y Biología Molecular, UNLP-CONICET)

- $\quad$ CINDEFI (Centro de Investigación y Desarrollo en Fermentaciones Industriales, UNLPCONICET)

- $\quad$ CREG (Centro Regional de Estudios Genómicos, UNLP)

- INIFTA (Instituto de Investigaciones Fisicoquímicas Teóricas y Aplicadas, UNLPCONICET)

- IFLP (Instituto de Física de La Plata, UNLP-CONICET)

- $\quad$ LSP (Laboratorio de Salud Pública, UNLP)

Integrantes del Grupo COVID Exactas (por orden alfabético de apellidos):

Leslie Amorós

Andres Angeletti

Monserrat Arciénaga

Paula Arrías
Emanuel Barbiera Romero

Cecilia Bergna

Julieta Bernengo

Alejandra Bosch
Fabiana Cabrera

Natalia Capriotti

Paula Carassi

Mariela Carrica 


\section{Mitids}

INNOVACIÓN Y DESARROLLO TECNOLÓGICO Y SOCIAL

Innovación y Desarrollo Tecnológico y Social (2020) 2 (2): 65-82- Número especial COVID-19

\begin{tabular}{|c|c|c|}
\hline Francisco Carriquiriborde & Micaela Iturralde & Federico Rasse Suriani \\
\hline Andrés Cordero & Luciana Juncal, & Mauricio Reynoso \\
\hline Agustina Corti & Yanina Lamberti & Gastón Rizzo \\
\hline Andrea Crivaro & Gabriel Lavorato & María E Rodríguez \\
\hline Renata Curciarello & Rolando Cristian Lillo & Claudia Rodríguez Torres \\
\hline Martina Debandi & M. Florencia López & David Romanín \\
\hline Laura Delaplace & Luciano Malaissi & Víctor Romanowski \\
\hline Elisa de Sousa, & Ivana Ivanoff Marinoff & Martín Rumbo \\
\hline Carolina Díaz & Elena Marson & Patricia L. Schilardi \\
\hline Guillermo H Docena & Flavia Massini & Lorena Tau \\
\hline Maia Elizagaray & Candela Masson & Carina Tersigni \\
\hline M. Leticia Ferrelli & Pedro Mendoza Zélis & Rosana Toro \\
\hline Cecilia Figoli & Juan Burgos Mignone & Soledad Traubenik \\
\hline Ulises Fleitas & María Laura Molina & Lucila Traverso \\
\hline Malena Ferreyra & Maria Victoria Nadalich & Julián Vaccaro \\
\hline Mariano Fonticelli & Sheila Ons & Hugo Valdez \\
\hline Fernando García Einschlag & Maximiliano Orezzo & Florencia Ventura \\
\hline Solange Giordano & Lisandro Petraglia & Carolina Vericat \\
\hline niela Hozbor & Matías Pidre & Mariano Volonté \\
\hline
\end{tabular}

María Ana Huergo Jimena Corbalán Quintana

- El Grupo COVID Exactas articula sus actividades con las Brigadas Sanitarias "Ramona Medina" (Secretaría de Extensión, Facultad de Ciencias Exactas, UNLP) 
Innovación y Desarrollo Tecnológico y Social (2020) 2 (2): 65-82- Número especial COVID-19

\section{Novedad u originalidad local en el conocimiento}

Con el conocimiento desde diciembre 2019 de los primeros casos de individuos infectados con un virus que produce una infección pulmonar (Wang, 2020), la identificación del virus emergente (Wuhan-Hu-1, GenBank: MN908947.1) y la posterior declaración de emergencia sanitaria y pandemia (22 de enero 2020 y 11 de marzo, respectivamente), la amenaza sanitaria se transformó en un desafío inédito para los sistemas sanitarios, económicos y socio-culturales de todos los países del planeta. Ante esta situación de crisis, distintos sectores de la comunidad han acometido un esfuerzo mancomunado para hacer frente a esta situación sanitaria inédita. En ese contexto colaborativo, en la Facultad de Ciencias Exactas se conformó el Grupo COVID Exactas a partir de distintos investigadores de diferentes Institutos y laboratorios de investigación para conformar un grupo de trabajo con dos objetivos:

1. Desarrollar metodologías para realizar diagnóstico, seguimiento o vigilancia

2. Apoyar al Laboratorio de Salud Pública (LSP), de la Secretaría de Extensión de la Facultad de Ciencias Exactas (FCE) en la implementación de la metodología existente y la desarrollada (Figura 1). 


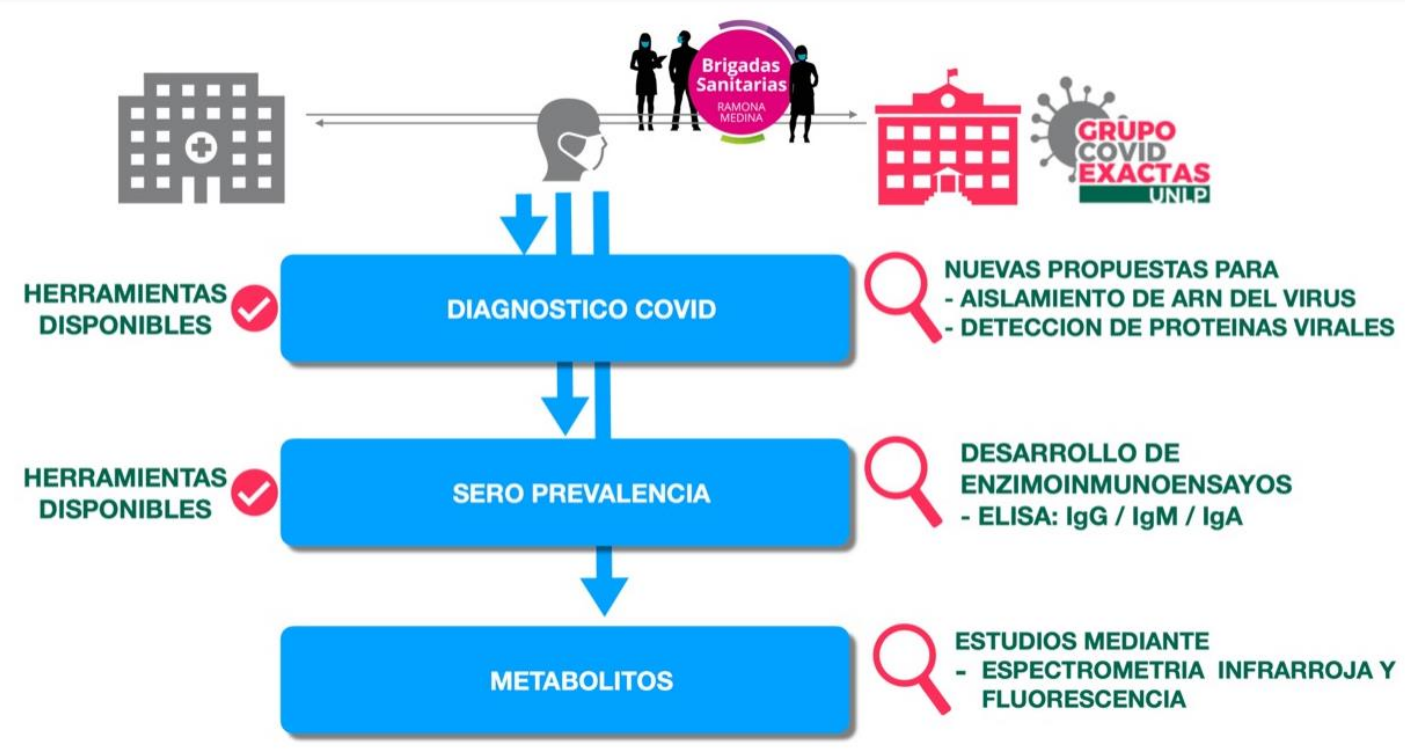

Figura 1. Esquema de trabajo del Grupo COVID Exactas para el fortaecimientos de las capacidad analítica del LSP.

Figure 1. Work scheme of the COVID Exactas Group for the strengthening of the analytical capacity of the LSP.

El 18 de marzo del 2020, el Presidente de la UNLP, Dr. Fernando Tauber, puso a disposición del Ministro de Salud de la Provincia de Buenos Aires las capacidades existentes en las distintas Facultades, para el eventual procesamiento de muestras humanas y diagnóstico de la infección por SARS-CoV-2. Así se conformó el Nodo de Diagnóstico de COVID-19 de la Universidad Nacional de La Plata (UNLP) con las Facultades de Ciencias Médicas, Ciencias Veterinarias y Ciencias Exactas y se firmó un convenio con el Ministerio de Salud de la Provincia de Buenos Aires para incorporarlo a la Red Nacional de Laboratorios de Influenza y otros virus 
Innovación y Desarrollo Tecnológico y Social (2020) 2 (2): 65-82- Número especial COVID-19

respiratorios. En este contexto, el Grupo COVID Exactas fue fundamental para proveer equipamiento y personal al LSP y, de esta manera, darle funcionalidad al laboratorio en el marco de la pandemia y de la respuesta que la UNLP estaba proponiendo a la comunidad y, en particular, a la región. Además, este grupo se abocó a la tarea de desarrollar métodos de detección de proteínas virales y anticuerpos específicos anti-SARS-CoV-2 para complementar las tareas de diagnóstico, seguimiento y vigilancia del LSP. Más adelante se inició la producción de nanopartículas magnéticas con el fin de desarrollar un método de extracción de RNA viral para facilitar la primera etapa del procesamiento de las muestras clínicas aportando un insumo de fabricación local de menor costo.

\section{Grado de relevancia}

El diagnóstico y la vigilancia epidemiológica constituyen aspectos fundamentales en el conocimiento de la enfermedad a medida que se desarrolla la historia natural de la infección por SARS-CoV-2. Además, constituyen herramientas esenciales para monitorear la circulación viral tanto en la actual situación como una vez que se implementen los planes de vacunación y en el período post-pandemia. Las metodologías aplicadas y desarrolladas en el LSP y en el Grupo COVID Exactas son herramientas importantes para cumplir con estos objetivos. Asimismo, el trabajo en conjunto con el Ministerio de Salud de la PBA, avalado por el Ministerio de la Producción de la Provincia de Buenos Aires, constituye un eje fundamental del 
Innovación y Desarrollo Tecnológico y Social (2020) 2 (2): 65-82- Número especial COVID-19

proyecto para coordinar tareas de monitoreo en forma conjunta, proveer información del estado inmune de pacientes infectados (donación de plasma de convalecientes) y definir medidas epidemiológicas sanitarias en el territorio provincial. De esta manera, la integración de la UNLP con instituciones gubernamentales y la comunidad constituye un pilar fundamental para intervenir en la resolución de esta problemática social/sanitaria existente. Para el desarrollo de esta propuesta se logró la conjunción de diferentes aportes de instituciones públicas y privadas, y al mismo tiempo se conformó en la FCE un grupo multidisciplinario de características singulares. El Grupo COVID Exactas fue fundamental para que el LSP iniciara sus actividades de diagnóstico, y actualmente ha generado un equipo de trabajo abocado a estudios serológicos con el fin de obtener información que complementa el diagnóstico, que permite definir la circulación viral y el estado inmune de individuos diagnosticados y no diagnosticados tanto de la población general y como del personal de Salud de establecimientos hospitalarios.

\section{Grado de pertinencia}

El Grupo COVID Exactas nuclea a virológos, biotecnólogos, microbiólogos, inmunólogos, físicos y matemáticos, mientras que el Laboratorio de Salud Pública (LSP), desde el año de su creación en la Facultad de Ciencias Exactas (2009), reúne a profesionales bioquímicos que han trabajado en el procesamiento y diagnóstico de 
Innovación y Desarrollo Tecnológico y Social (2020) 2 (2): 65-82- Número especial COVID-19

muestras de la pandemia H1N1 y luego continuó trabajando en el Programa de HIV de la UNLP. De esta manera, el conjunto de profesionales del LSP, en el contexto del Grupo COVID Exactas, conjuga experiencias y capacidades de un número muy importante de docentes-investigadores, estudiantes y personal de apoyo de modo de potenciar un trabajo multidisciplinario frente a la emergencia sanitaria actual. Se espera que este trabajo conjunto entre profesionales bioquímicos e investigadores refuerce la capacidad de diagnóstico, seguimiento y vigilancia de la COVID-19 durante la pandemia, y en las etapas posteriores a la misma. La consolidación de esta actividad conjunta será fundamental para mantener la vigilancia territorial junto al control de la enfermedad, y para el control poblacional en la etapa postvacunación. En este contexto, la integración de la Universidad Nacional de La Plata, en este caso a través de la Facultad de Ciencias Exactas, con el Ministerio de Salud de la Provincia de Buenos Aires y el Ministerio de Producción de la Provincia de Buenos Aires, constituye un hito fundamental para contribuir a la resolución de problemas sociales y sanitarios regionales.

\section{Grado de demanda}

La pandemia desde sus inicios ha generado una intensa demanda de diagnóstico específico, que actualmente se desarrolla en el LSP. Al comienzo, el principal requerimiento fue montar el laboratorio diagnóstico y la aplicación de la técnica de RT-qPCR para detectar genes virales en muestras de hisopados de vías respiratorias 
Innovación y Desarrollo Tecnológico y Social (2020) 2 (2): 65-82- Número especial COVID-19

provenientes de individuos sospechosos de estar infectados. Sin embargo, a medida que la pandemia fue avanzando, surgió una creciente necesidad de estudiar la presencia de anticuerpos (serología) sobre muestras de sangre. Además de la identificación de individuos infectados, la serología actualmente es esencial para conocer la circulación viral, la seroconversión de pacientes COVID-19 positivos, la identificación de individuos que han sido asintomáticos o no diagnosticados y que han estado expuestos al SARS-CoV-2 (complemento del diagnóstico), y finalmente la evaluación de la seroconversión en pacientes que, luego de haber curado de la infección, están en condiciones de ser donantes de plasma. En etapas posteriores, una demanda adicional indudablemente será la del monitoreo poblacional de anticuerpos específicos post-vacunación, como marcador de una respuesta inmune específica, y de perduración de la protección inmune en el tiempo. En todos estos aspectos el trabajo permanente con diferentes autoridades del Ministerio de Salud de la Provincia de Buenos Aires ha resultado esencial para planificar y coordinar distinto tipo de acciones en los ejes diagnóstico y vigilancia. En este escenario desde el LSP se han generado acciones tendientes a cubrir estos aspectos, y al mismo tiempo, desde el Grupo COVID Exactas, se ha generado un grupo de trabajo abocado al desarrollo de métodos inmunoenzimáticos para la detección de antígenos virales o anticuerpos específicos.

Al mismo tiempo, algunos miembros del Grupo COVID Exactas son parte de los grupos de asesores del Ministerio de Salud de la Provincia de Buenos Aires, 
Innovación y Desarrollo Tecnológico y Social (2020) 2 (2): 65-82- Número especial COVID-19

Ministerio de Salud de Nación, Gobernación de la Provincia de Buenos Aires y del Comité Técnico para el Diagnóstico de SARS-CoV-2 de la UNLP. La intervención de miembros del LSP y del Grupo COVID en medios de difusión también ha sido un aporte relevante (diarios, programas de televisión, comunicados desde las distintas Sociedades Científicas, asociaciones profesionales, webinars y otros medios de difusión).

\section{Desarrollo de metodologías analíticas para diagnóstico, seguimiento y}

\section{vigilancia epidemiológica}

A través de esta estrategia de fortalecimiento de las capacidades operativas del Laboratorio de Salud Pública de la Facultad de Ciencias Exactas de la Universidad Nacional de La Plata, el Grupo COVID Exactas está desarrollando distintos métodos para la detección de antígenos virales (proteína N o polipéptido RBD de Receptor Binding Domain que corresponde a la porción de la proteína S que se une al receptor ACE2), anticuerpos séricos específicos (IgG, IgM e IgA específicos de $\mathrm{N}$ o RBD) y metabolitos en orina. Estas diferentes metodologías pueden ser empleadas con distintos fines: complementar el diagnóstico, realizar vigilancia en barrios y en Personal de Salud de establecimientos hospitalarios, el monitoreo de la respuesta inmune y progreso de la enfermedad en pacientes internados COVID positivos, y para el control de protección inmune en individuos convalecientes o luego de vacunados. Actualmente, se está empleando el kit COVIDAR, provisto por el 
INNOVACIÓN Y DESARROLLO TECNOLÓGICO Y SOCIAL

Innovación y Desarrollo Tecnológico y Social (2020) 2 (2): 65-82- Número especial COVID-19

Ministerio de Salud de la Provincia de Buenos Aires, para detectar anticuerpos IgG específicos de la proteína $S$ y RBD y se han analizado hasta el momento aproximadamente 2800 muestras (Personal de Salud, vigilancia y pacientes internados COVID positivos) (Tabla 1).

Tabla 1. Muestras analizadas a octubre de 2020. Serología por COVIDAR, RT-qPCR y espectroscopía de fluorescencia e infrarrojo de muestras de orina.

Table 1. Samples analyzed as of October 2020. Serology by COVIDAR, RT-qPCR and fluorescence and infrared spectroscopy of urine samples.

\begin{tabular}{|l|c|c|c|}
\hline Determinación & Positivos (n) & \% positivos & Negativos (n) \\
\hline SEROLOGIA-Salud & 90 & 10,4 & 91 \\
\hline SEROLOGIA-Hospitales & 175 & 48,1 & 189 \\
\hline SEROLOGIA-Vigilancia & 86 & 14,7 & 499 \\
\hline RT-qPCR/Hisopados & 7400 & 38,9 & 11600 \\
\hline Espectroscopía/Orinas & 170 muestras en estudio \\
\hline
\end{tabular}

Salud corresponde a personal de Salud de hospitales y UPA; Hospitales corresponde a pacientes internados COVID positivos; Vigilancia corresponde a individuos de barrios de La Plata y Gran La Plata; Orinas corresponde a muestras de pacientes COVID positivos internados; RT-qPCR/Hisopados: corresponde a muestras de individuos con sospecha clínica de COVID-19.

El diagnóstico de la COVID-19 se realiza en el LSP a través de la metodología de referencia RT-qPCR empleando kits comerciales e insumos provistos por el Ministerio de Salud de la Provincia de Buenos Aires y la Universidad Nacional de La 
Innovación y Desarrollo Tecnológico y Social (2020) 2 (2): 65-82- Número especial COVID-19

Plata. Hasta el momento se han analizado más de 19.000 muestras provenientes de distintas regiones sanitarias de la Provincia de Buenos Aires. Para apoyar la actividad cotidiana del LSP y de otros laboratorios de la PBA, se articuló el conocimiento y la capacidad de grupos de trabajo en física (IFLP), fisicoquímica (INIFTA) y biología molecular (IBBM y CREG) para montar un método de extracción de RNA viral basado en el aprovechamiento de nanopartículas magnéticas. Este método evita el uso de centrífugas, simplificando el procedimiento. Por otra parte, el desarrollo de un método de detección de antígenos virales permitirá contar con una metodología complementaria de diagnóstico, basada en la interacción antígeno-anticuerpo a partir de diferentes muestras biológicas. Esta metodología llevada a un formato point-of-care ( $P O C$, al lado del paciente) permitirá hacer relevamiento diagnóstico a campo y se complementará con el método de biología molecular de referencia, o la técnica de amplificación isotérmica. Asimismo, se evaluará la aplicación de esta metodología para la detección de antígenos virales en muestras ambientales (Tabla). Asimismo, el LSP ha participado en ensayos de validación de distintos métodos (Neokit, Chemtest, ensayos de detección rápida de antígenos, etc.).

Por otro lado, los métodos inmunoenzimáticos que permiten detectar la presencia de anticuerpos específicos en sangre periférica complementan el diagnóstico de la patología principalmente en pacientes no hisopados, con resultados falsos negativos y que hayan tenido una clínica compatible, o en individuos asintomáticos. 
Innovación y Desarrollo Tecnológico y Social (2020) 2 (2): 65-82- Número especial COVID-19

Estas determinaciones permiten obtener información sobre la circulación viral, la duración de la inmunidad adquirida post-infección, si se trata de pacientes en los que no se produjo seroconversión durante la infección natural, considerar la posibilidad de re-infección en pacientes sin seroconversión, la identificación de individuos que fueron COVID positivos y estarían en condiciones de donar plasma, y la generación de inmunidad protectora post-vacunación, como así también su persistencia en el tiempo.

En la vigilancia epidemiológica se están aplicando estos métodos (kit COVIDAR) para el monitoreo de la seroconversión en barrios de La Plata y Gran La Plata (Barrios José Luis Cabezas y Villa Argüello) en un trabajo en conjunto con la Secretaría de Extensión de la Facultad de Ciencias Exactas a través del trabajo de campo de las Brigadas Sanitarias Ramona Medina, Región Sanitaria XI y los Municipios de Ensenada y Berisso. Por otro lado, se está realizando el monitoreo de personal de Salud de diferentes hospitales y lugares de atención primaria (Hospital Italiano de La Plata, Hospital de Lezama, distintas UPA). La identificación de población infectada, con y sin activación del sistema inmune se complementa con el trabajo de monitoreo del Ministerio de Salud de la Provincia de Buenos Aires. Es importante monitorear a los pacientes internados durante la evolución de la enfermedad e identificar a aquellos en los que se verifica una seroconversión, caracterizar los niveles de anticuerpos en función de la severidad de la patología o de los tratamientos recibido. Asimismo, se apunta a seguir los niveles de anticuerpos en 
Innovación y Desarrollo Tecnológico y Social (2020) 2 (2): 65-82- Número especial COVID-19

aquellos pacientes que recibieron plasma de convalecientes, y finalmente, los niveles de anticuerpos al momento del alta hospitalaria. En pacientes enfermos e internados es importante este dato para complementar el cuadro clínico y establecer correlaciones entre la evolución de su carga viral, la clínica y el progreso de su estado inmune (Tabla).

Además, los grupos de espectroscopía de fluorescencia del INIFTA y de espectroscopía infrarroja del CINDEFI están trabajando en la detección espectroscópica en muestras de orina de determinados metabolitos como indicadores de pronóstico de la enfermedad o de evolución clínica de la misma. Las muestras que se están estudiando actualmente provienen de la población general, de barrios populares, y de pacientes hospitalizados. Los espectros tanto de fluorescencia como infrarrojo proporcionan una "huella digital molecular" que representa la composición bioquímica y estructural de las muestras de orina. Dada la gran cantidad de información contenida en las señales registradas, las bandas/picos presentes en cada espectro se analizan mediante la aplicación de métodos quimiométricos, empleando diversas técnicas estadísticas $y / o$ de matemáticas avanzadas. Hasta el momento se han obtenido 170 muestras de orina y se han registrado sus respectivos espectros infrarrojos y de fluorescencia. A partir del reconocimiento de los perfiles de estos espectros se buscará extraer información de relevancia que nos permitiría monitorear la evolución de la infección. A través de estos estudios se pretende también correlacionar los cambios 
Innovación y Desarrollo Tecnológico y Social (2020) 2 (2): 65-82- Número especial COVID-19

espectrales con parámetros serológicos y con los indicadores, tanto clínicos como bioquímicos, de progreso de la enfermedad.

En conclusión, a partir de una necesidad regional en el contexto de la crisis sanitaria determinada por la pandemia de COVID-19, la Facultad de Ciencias Exactas articuló en forma inédita y en muy corto tiempo la conformación de un grupo multidisciplinario, el Grupo COVID Exactas, que definió y montó el Laboratorio de Virología en el Laboratorio de Salud Pública, para luego articular con la Universidad Nacional de La Plata y el Ministerio de Salud de la Provincia de Buenos Aires, su incorporación a la Red Nacional de Diagnóstico de COVID-19. Al mismo tiempo se generó un proyecto para desarrollar metodologías que fortalezcan al LSP en diagnóstico, seguimiento de la respuesta inmune, y monitoreo y pronóstico de la enfermedad. Esta conjunción de actividades no sólo significa un aporte durante la pandemia, sino que será esencial en momentos post-pandemia para vigilancia poblacional y testeo de la inmunidad adquirida en individuos vacunados.

\section{Información sobre el patentamiento/registro de la innovación o desarrollo}

Si bien se va a desarrollar tecnología con potencialidad de protección intelectual, este aspecto aún no ha sido implementado. 


\section{Financiamiento}

Se ha recibido financiación para el desarrollo del presente proyecto de la Universidad Nacional de La Plata, de la Facultad de Ciencias Exactas y del Ministerio de Ciencia, Tecnología e Innovación (Proyecto BsAs 47 COVID). Asimismo, se han recibido donaciones de la Fundación Bioquímica Argentina. Uno de los productos en desarrollo ha sido motivo de un convenio tripartito entre la PBA, la UNLP y el CONICET.

\section{Agradecimientos}

Agradecemos a las siguientes instituciones por el apoyo recibido: Universidad Nacional de La Plata, Facultad de Ciencias Exactas, Ministerio de Salud de la Provincia de Buenos Aires, Ministerio de Producción de la Provincia de Buenos Aires, Fundación Bioquímica Argentina, Colegio de Bioquímicos de la Provincia de Buenos Aires y Centro Científico Tecnológico CONICET de La Plata. Un agradecimiento especial a los pacientes que firmaron los consentimientos informados para ceder sus muestras a este proyecto y a todos los médicos (entre ellos el Dr Jorge Milone del Hospital Italiano de La Plata, Dra Cecilia Bergna del Hospital San Juan de Dios de La Plata y Dra Karina Aguilera del Sanatorio IPENSA), bioquímicas (Bioq. María E. Braviz López del Hospital San Juan de Dios de La Plata y Bioq. Victoria Maydana del Sanatorio IPENSA) y demás personal de Salud que de una u otra manera colaboran en este proyecto. 


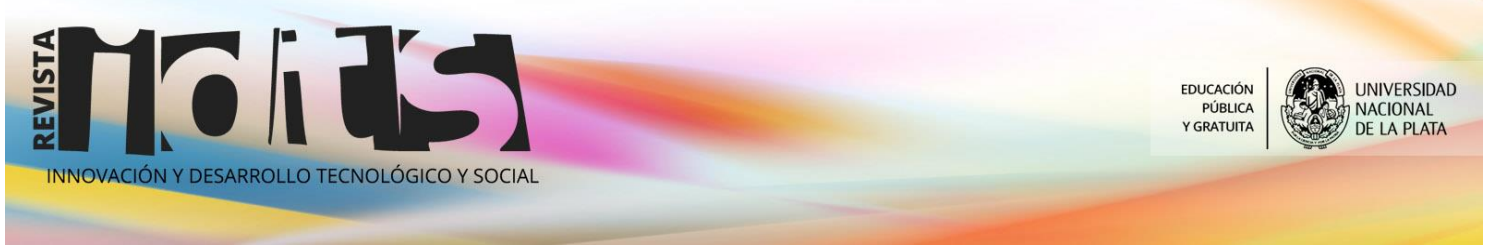

Innovación y Desarrollo Tecnológico y Social (2020) 2 (2): 65-82- Número especial COVID-19

\section{Referencias bibliográficas}

Wang, D., Hu, B., Hu, C., Zhu, F., Liu, X., Zhang, J., Wang, B., Xiang, H., Cheng, Z., Xiong, Y., Zhao, Y., Li, Y., Wang, X., \& Peng, Z. (2020). Clinical Characteristics of 138 Hospitalized Patients With 2019 Novel Coronavirus-Infected Pneumonia in Wuhan, China. JAMA, 323(11), 1061-1069. https://doi.org/10.1001/jama.2020.1585

Wuhan seafood market pneumonia virus isolate Wuhan-Hu-1, complete genome. GenBank: MN908947.1 https://www.ncbi.nlm.nih.gov/nuccore/MN908947.2 